\title{
CypherDB: A Novel Architecture for Outsourcing Secure Database Processing
}

\author{
Bony H. K. Chen, Paul Y. S. Cheung, Peter Y. K. Cheung, and Yu-Kwong Kwok
}

\begin{abstract}
CypherDB addresses the problem of protecting the confidentiality of database stored externally in a cloud and enabling efficient computation over it to thwart any curious-but-honest cloud computing service provider. It works by encrypting the entire outsourced database and executing queries over the encrypted data using our novel CypherDB secure processor architecture. To optimize computational efficiency, our proposed processor architecture provides tightly-coupled datapaths that avoid information leakage during database access and query execution. Our simulation using a well-known database benchmark TPC-H over a commercial grade Database Management System (SQLite) demonstrates that our proposed architecture incurs an average of about $10 \%$ overhead when compared with the same set of operations without secure database processing.
\end{abstract}

Index Terms—Database Security, Secure Processor, Computer Architecture, Cloud Security, Hardware Security, Confidentiality

\section{INTRODUCTION}

D ATABASE-AS-A-SERVICES (DBaaS), one of the rapidly emerging services in Cloud Computing, provides database outsourcing, database administration and query execution services to the client. However, one of the biggest barriers toward dependable cloud computing is the security concern. As revealed by a global security study [1], more than half of the participants rated data security as their major consideration of storing their data in a cloud.

A major deficiency in cloud security is that user data is stored in third-party servers in plaintext. Although it is assumed that the cloud service provider can be trusted by a contractual agreement, it does not necessarily imply that the server administrators are equally trustworthy. A recent security breach in Google [2] shows that any server administrator with access to the confidential information can abuse it at ease. An even worse issue is that an honest-but-curious server administrator is capable of performing massive breach of privacy without being detected. One obvious solution to protect against such attack is by encrypting the outsourced database without revealing the encryption key to the administrator. However, it poses one daunting challenge: the computation on an encrypted data without the encryption key is at best very inefficient, or at worst impossible.

To address this security problem, there have been

- Bony H. K. Chen is with both the Department of Electrical and Electronic Engineering of the University of Hong Kong and Imperial College London. Email: h.chen11@imperial.ac.uk

- Paul Y. S. Cheung and Yu-Kwong Kwok are with the Department of Electrical and Electronic Engineering, The University of Hong Kong. Pokfulam Road. Hong Kong.

E-mail: paul.cheung@hku.hk; ricky.kwok@hku.hk

- Peter Y. K. Cheung is with the Department of Electrical and Electronic Engineering, Imperial College London. SW7 2AZ London. U.K.

Email:p.cheung@imperial.ac.uk many proposed solutions: using homomorphic encryption [3], [4], [5] or using a trusted coprocessor [6], [7]. However, they are either incomplete or infeasible (with low efficiency). In this paper, we propose a novel cloud system architecture called CypherDB, which is based on re-designing the processor architecture to support arbitrary computation on encrypted data. Our design tightly couples database encryption with our proposed architecture. With our method, the encrypted data from the database can be operated in our novel processors in a distributed and parallel manner. Our main contributions are as follows:

- To the best of our knowledge, CypherDB is the first to use processor architectural design to successfully protect remote operation on encrypted database against any honest-but-curious administrator.

- We introduce a novel approach to encrypt the database that allows database operation to be performed efficiently and securely on CypherDB secure processor in a distributed database system.

- We propose a run-time memory partitioning system to secure on-the-fly execution with a novel use of software-transparent cache line encryption and a per query random key.

- We show that CypherDB, being able to support arbitrary database operations, requires only a minimal software changes and achieves well performance with only $10 \%$ performance overhead on average.

The remainder of the paper is structured as follows. Section 2 outlines the CypherDB cloud execution model. Section 3 describes the database encryption strategies. Section 4 describes the design of our novel processor architecture. Section 5 presents the experiments conducted and the performance evaluation. Section 6 provides a security analysis to our solution. Section 7 describes related work. Section 8 concludes the paper. 


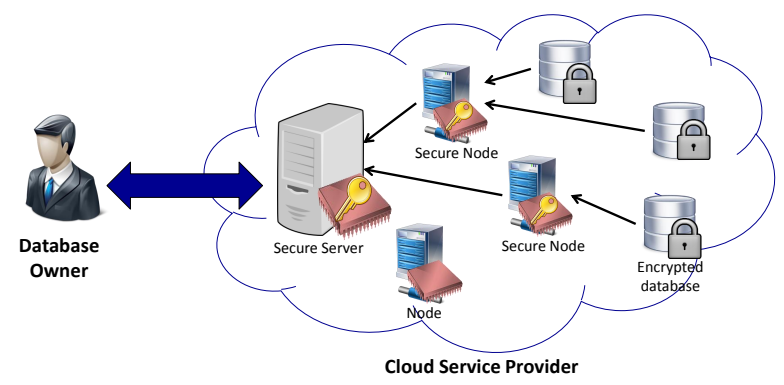

Fig. 1: CypherDB application scenario.

\section{CypherDB Overview}

Figure 1 depicts an application scenario of our CypherDB cloud model, which involves two parties: a cloud service provider (CSP) and a database owner. The database owner exports an encrypted database to the CSP for future querying. The CSP hosts the database server and provides storage and database administration service to the database owner.

To process the encrypted database, a server/node in CSP has to be "empowered" with two features: 1) equipping a CypherDB secure processor and, 2) having the database encryption key stored inside the processor chip. We refer these "empowered" servers/nodes as secure servers/nodes. A normal server/node (without neither CypherDB secure processor nor database encryption key) is not capable of processing the encrypted database. To query the outsourced database, the database owner communicates with a single secure server as if the entire database is stored in it. In CSP, outsourced encrypted database is partitioned and stored in a distributed manner, whereas the secure server manages the query processing on such distributed database.

\subsection{Architectural Model of a Secure Server/Node}

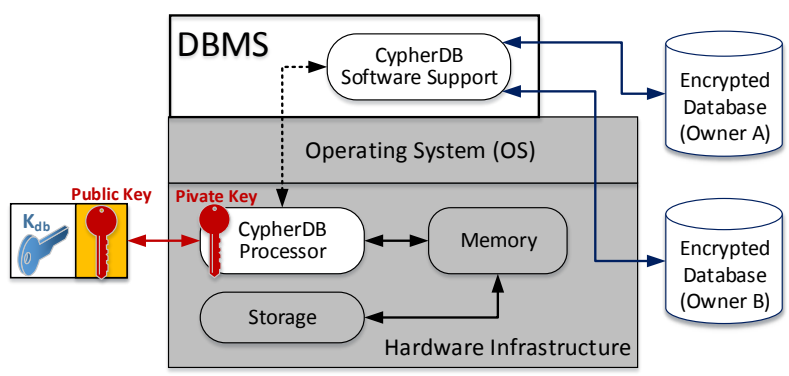

Fig. 2: Architectural mode of each secure server/node. Components in grey represent untrusted entities while those in white represent trusted entities.

Each CypherDB secure processor has a unique private key burned into the processor chip and is not accessible to any software. Database encryption key $K_{d b}$ can thus be sent to the processor chip through key encapsulation encrypting $K_{d b}$ with the public key of the processor and decrypting it using the on-chip private key [8]. Such key exchange mechanism allows different database owners

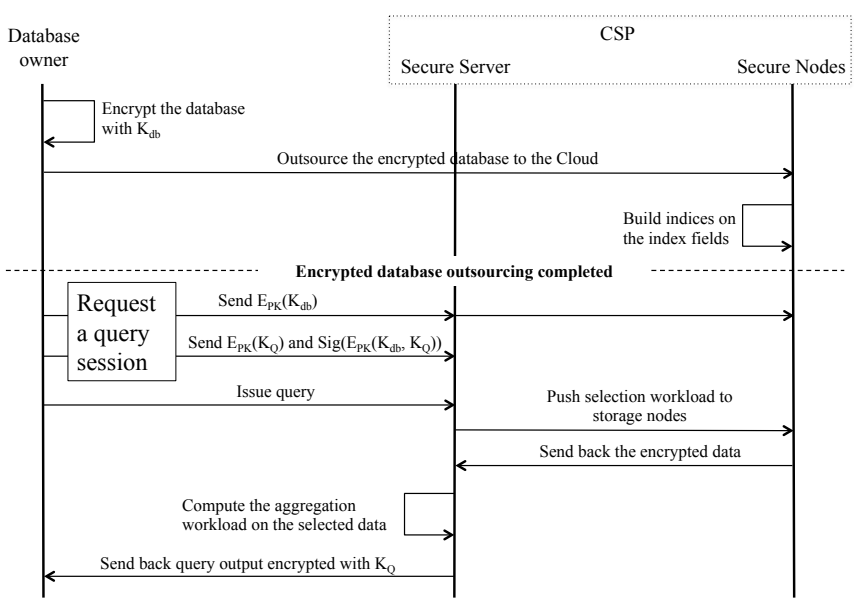

Fig. 3: CypherDB execution flow.

to send their $K_{d b}$ to a single secure server/node. Therefore, secure servers/nodes can process the encrypted database from any database owners as far as their corresponding $K_{d b}$ is submitted.

Figure 2 depicts the architectural model of a secure server/node. The DBMS, running on top of the host operating system (OS), can process the encrypted databases from different database owners (with the corresponding $K_{d b}$ stored inside the secure processor). The CypherDB software support, which is embedded into the DBMS, can talk to the secure processor in order to perform some security-critical tasks (e.g. submitting the encapsulated $K_{d b}$ to the processor, triggering hardware support to process encrypted data).

\subsection{Execution Flow}

Figure 3 depicts the execution flow of outsourcing and querying the encrypted database outsourcing. The database owner first encrypts the database with $K_{d b}$ and specifies the columns to be built as indices before outsourcing it to the CSP. The distributed encrypted database is managed by multiple secure nodes whereas the indices are built accordingly.

To query the outsourced database, the database owner can set up a secure query session by sending $K_{d b}$ and a query encryption key $K_{Q}$ to the allocated CypherDB secure processors. In brief, it involves the following procedures:

1) The database owner requests a secure query service from CSP and specifies the resources needed (e.g. number of cores, bandwidth).

2) The CSP allocates the resources as requested and sends all the public keys $\left\{P K_{i}\right\}$ of the secure processors involved to the database owner.

3) The database owner encrypts $K_{d b}$ with all public keys. $K_{Q}$ is only encrypted with the public key of the secure server $P K_{C}$ he talks to. He further signs a digital signature $\operatorname{Sig}\left(E_{P K}\left(K_{d b}, K_{Q}\right)\right)$ that binds $K_{d b}$ and $K_{Q}$ together to be verified by the CypherDB software support. 
4) The database owner sends all encrypted database encryption key $\left\{E_{P K_{i}}\left(K_{d b}\right)\right\}$, query encryption key $E_{P K_{C}}\left(K_{Q}\right)$ and the digital signature $\operatorname{Sig}\left(E_{P K}\left(K_{d b}, K_{Q}\right)\right)$ to the CSP.

5) The CSP distributes $\left\{E_{P K_{i}}\left(K_{d b}\right)\right\}$ to the appropriate secure processors in order to store $K_{d b}$ securely on-chip. It further verifies $\operatorname{Sig}\left(E_{P K}\left(K_{d b}, K_{Q}\right)\right)$ and stores $K_{Q}$ on the secure processor chip of the secure server.

Upon receiving the query from the database owner, the secure server first pushes the selection workload to the pre-allocated secure nodes. After that, the secure nodes send back a set of relevant encrypted records. The secure server will then compute the final join or aggregation workload. Finally, the query output is encrypted with $K_{Q}$ before returning to the database owner.

\subsection{Database Virtualization}

We envision that the CSP could set up a collection of servers/nodes equipped with CypherDB secure processors, which forms a secure cluster. Resource pooling can thus be performed in the secure cluster for secure query processing. We address four important virtualization features that may be potentially impacted by our proposed solution, which are discussed as follows:

Resource Allocation: Since each CypherDB secure processor can accommodate the database encryption keys from different database owners, the CSP can allocate any available secure server/nodes, following the key exchange procedure described in Section 2.2, to the database owners upon a request of secure query session.

Resource Re-allocation: Migration of process may be necessary due to load balancing and fault tolerance in the Cloud (i.e. move the query processing process from one secure server/node to another). In this case, the CSP can re-allocate some secure server/nodes and send the corresponding secure processors' public keys to the database owner for exchanging the database encryption key to those "new" secure server/nodes. On the other hand, database owner may also request for more resource by repeating the secure query session request described in Section 2.2.

Distributed Storage: In CypherDB, the database is encrypted in logical level that eases the partitioning process. Horizontal partitioning (i.e. in a record basis) can be performed directly on the encrypted database without involving any re-encryption.

Multi-tenancy: CypherDB secure processor contains an on-chip memory for key storage that can accommodate multiple database and query encryption keys from various database owners. CypherDB software support is responsible for managing the multi-tenant execution environment and switching the use of the onchip database/query encryption key accordingly so as to support multi-tenant query processing on a single server/node.

\subsection{Threats and Assumptions}

We assume the CSP in our model is honest-but-curious. In general, it obeys any communication protocol agreed with the database owner and deploys database operations correctly. Any detectable tampering can be protected by a contractual agreement between them. However, this cannot protect any passive attack leaving no trace on the system (i.e. read/copy data from storage disk, main memory or probe processor-to-memory data bus). The goal of an adversary is to read the content of the database without being detected. In summary, we consider the following threats and assumptions:

- An adversary can launch a malicious process or virtual machine (VM) or even get access to the OS layer to copy or read the data in off-chip memory. He can also probe the memory bus to read the data in processor-to-memory traffic.

- The communication channels between the database owner and secure server or among secure servers and secure nodes are all open and subject to eavesdropping.

- CypherDB processor is assumed to be the only trusted hardware in the system. Other off-chip hardware components are assumed to be malicious.

- The DBMS (with CypherDB software support) is assumed to be securely booted by employing secure boot technology (e.g. Bastion [9], TPM [8]). Run-time Execution Validator [10] can also be employed to ensure that the DBMS is running as expected.

- Side-channel attacks such as timing-attack or poweranalysis attack are not considered in this paper because these attacks are prohibitively costly to implement in a typical data center environment. Indeed, to launch these attacks, the attacker needs to breach physical security of the data center and this is highly unlikely to happen. Any hardware tampering on the processor is also assumed to be infeasible in the cloud environment.

\section{Database Encryption}

In this section, we discuss how the database owner encrypts the database before outsourcing it to the Cloud. The database is encrypted in logical level to allow scalable and parallel query processing. The choice of encryption scheme is to allow efficient processing in our CypherDB secure processor. The entire database is protected by encrypting each field of a database table. We refer a field and a row of a database table as attribute and record respectively in the rest of the paper.

\subsection{Attribute Encryption}

Each attribute is encrypted with AES in one of two modes: 1) counter mode (AES-CTR) or, 2) outputfeedback mode (AES-OFB). These two encryption modes 


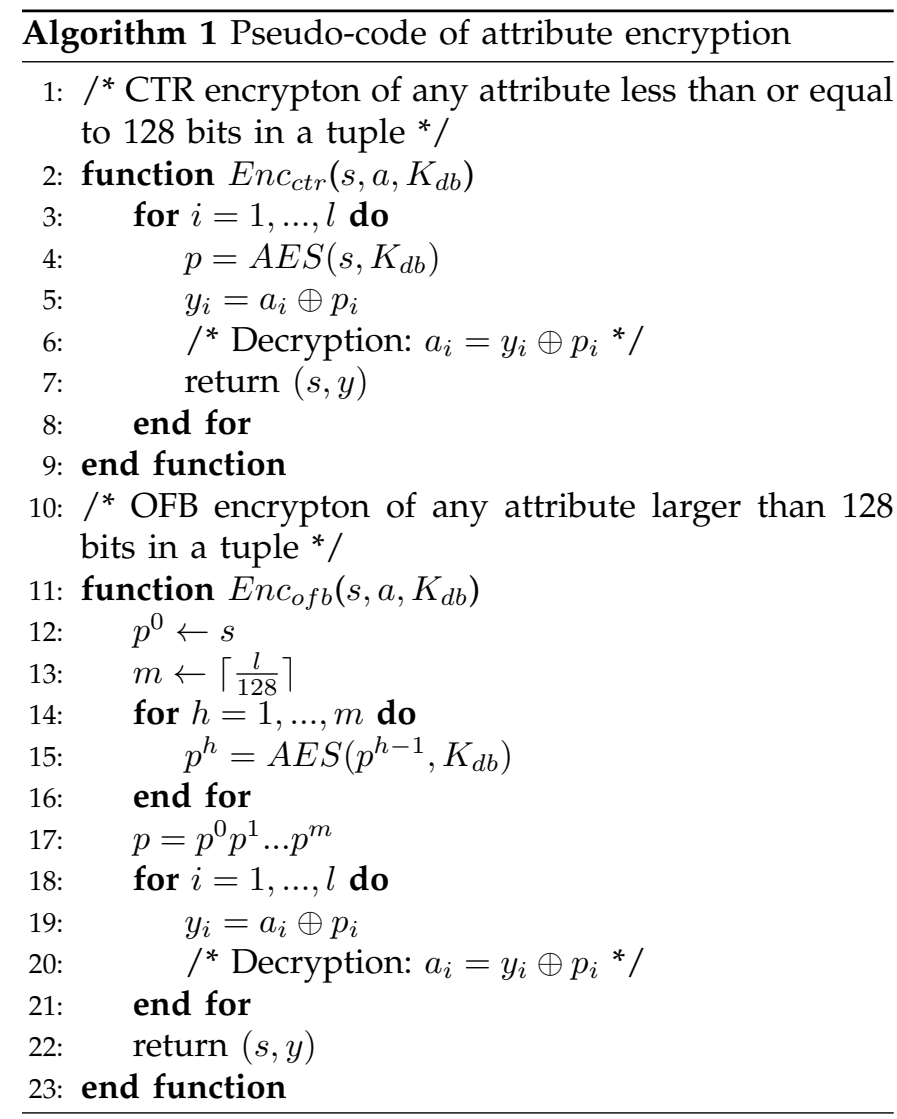

are chosen due to two important objectives: 1) offloading the encryption/decryption work, and 2) transforming block cipher into stream cipher, which are favourable in our processor design.

Algorithm 1 explains the operation of these two encryption modes in pseudo-code. AES-CTR encrypts any attribute less than or equal to 128 bits. The function $E n c_{c t r}$ encrypts the l-bit long attribute, denoted as $a$, using the 128-bit attribute seed $s$ and database key $K_{d b}$. The encryption is done by XOR-ing the most significant $l$ bits of the encryption pad with the data (line 3-5). To encrypt the attribute longer than 128 bits, AES-OFB function $E n c_{o f b}$ generates a series of 128-bit encryption pads to be encrypted with the data (line 14-16). The most significant $l$ bits of the concatenated encryption pad $p^{0} p^{1} \ldots p^{m}$ is then used to encrypt the attribute data (line 17-19). Decryption is done by XOR-ing the ciphertext with the same encryption pad (line 6 and 20). Note that AES-CTR and AES-OFB both use AES encryption but in different operation modes (line 4 and line 15).

\subsection{Attribute Encryption Seed}

The challenge of encrypting the attribute with AES-CTR and AES-OFB is to maintain the uniqueness of the seed $s$ under the same database key $K_{d b}$ [11]. In other words, each attribute across a database must own a distinct seed to each other (spatial uniquess), whereas the seed for the same attribute must not repeat for every update operation on that attribute (temporal uniqueness).
Despite the security concern, the choice of encryption seed can also result in performance problem. If we simply assign a random 128-bit unique seed to each 32-bit/64-bit attribute, it not only introduces $5 \times / 3 \times$ database storage overhead, but also increase the program execution time because these encryption seeds have to be processed (to generate the encryption pad) for the actual data encryption/decryption.

\subsubsection{Seed Components}

Due to these security and performance concerns, our design is to leverage the logical schema of the database to formulate the seed. In the structure of a logical schema, if each element of the schema has its own identifier (ID), each attribute can be identified by (databaseID, tableID, rowID, columnID) which is spatially unique across various databases and tables. Temporal uniqueness can be achieved by appending a global incremental counter cntr to each record, which is shared by each attribute within that record. Although having a record counter introduces a penalty on re-encrypting the non-updated attribute within that record, it is chosen due to two reasons. First, it can significantly reduce seed overhead because the counter cntr is not an element in the logical schema which has to be stored externally. Second, this penalty is insignificant on any analytical data mining workload because such workload is readintensive rather than write-intensive.

\subsubsection{Seed Formulation}

In a typical database application, logical schema is used in most operating layers and is eventually translated into its physical schema in order to locate the record in the database file. The formation of the attribute seed can thus embedded into the logical-to-physical schema translation software process. In other words, the actual program execution is able to "generate" the encryption seeds by re-using some software execution parameters, at run-time. Take SQLite as an exmaple. The address of an attribute in a record is stored in an array $a O f f-$ Set[ColumnID] where ColumnID is the index. This index is thus used as one of our attribute seed components. This approach has two main advantages: 1) significantly reduces the storage overhead and ,2) introduces minimal software execution overhead. Only the record counter cntr $_{i}$ needs external storage and software management.

\subsection{Index Protection}

Encrypting the attribute with AES in either CTR or OFB mode prohibits the $\mathrm{B}^{+}$-tree indexing, which is one of the most commonly used indexing strategies in database system. To allow remote indexing and protect the indices at the same time, we adopt order-preserving encryption (OPE) to encrypt the indices in the same way as suggested in [12].

OPE is an encryption scheme that can perform order operations on ciphertexts in the same way as plaintexts 


\begin{tabular}{|c|c|c|c|c|}
\hline ROW ID & ID & Name & Remark & \\
\hline 3 & $\begin{array}{c}16 \\
\mid \\
(2,2,3,1)\end{array}$ & $\begin{array}{c}\text { Alex } \\
\quad \text { । } \\
(2,2,3,2)\end{array}$ & $\begin{array}{r}\text { He is ... } \\
(2,2,3,3)\end{array}$ & \\
\hline $\mathrm{K}_{\mathrm{db}}$ & AES-CTR & AES-CTR & AES-OFB & OPE \\
\hline ROW ID & $\begin{array}{c}\downarrow \\
\text { ID }\end{array}$ & $\begin{array}{c}\downarrow \\
\text { Name }\end{array}$ & $\begin{array}{c}\downarrow \\
\text { Remark } \\
\end{array}$ & $\begin{array}{c}\text { Index field } \\
\downarrow \\
\mathrm{ID}_{\mathrm{OPE}}\end{array}$ \\
\hline 3 & 0x28abdc72 & $0 \times 46 c 17 a 6 e$ & $0 \times 87 c 3 f d 1 \ldots$ & 23738492 \\
\hline
\end{tabular}

Fig. 4: An example showing the how the database is encrypted before outsourcing.

(i.e. $\operatorname{Enc}(x)>\operatorname{Enc}(y)$ iff $x>y)$ and is well proven to reveal no additional information about the plaintext values beside their order [12].

The use of OPE to encrypt the indices can achieve two important objectives: 1) to allow remote indexing in the cloud for scalability and elasticity, and 2) to leverage the order-preserving property of OPE for efficient B-tree index search because decryption is not required for range check operation.

\subsection{An Illustrative Example}

Figure 4 shows an example of a record of database table containing three attributes in a database table. The attribute longer than 128 bits (Remark) is encrypted with AES-OFB, and otherwise (ID, Name), encrypted with AES-CTR using $K_{d b}$. Each corresponding attribute seed is shown in the blanket in the format of (databaseID, tableID, rowID, columnID). Assume the attribute $I D$ is used as an index, it is further encrypted by OPE and the output $I D_{O P E}$ is appended to the encrypted tuple. While the encrypted tuple is sent to the Cloud, the column $I D_{O P E}$ is used directly (without decryption) to create the indices in the Cloud. The encrypted record is stored according to the storage strategy of the DBMS.

\subsection{Remote Database Modification}

As the database can be dynamically changing over time, a mechanism to support remote modification of the encrypted database is necessary. The major challenge in CypherDB is that users can easily lose track of the attribute seeds being used in encrypting the data. To overcome this challenge, a dynamic database attribute seed management is developed to ensure the uniqueness of the attribute seed used. The key idea is to let users do a bookkeeping on the attribute seed table.

For any database management system, CREATE TABLE, INSERT, DELETE and UPDATE are the most commonly used operations in modifying any database. We therefore discuss the working principles of the attribute seed management under these operations.

CREATE TABLE: To create a database table, users can issue the CREATE TABLE query statement as normal. The cloud server then needs to send back the corresponding databaseID and tableID to the users so that they can be used to encrypt the new records.
INSERT: To insert an encrypted record to the outsourced database, it involves the following 3 steps procedures: 1) chooses an unused rowID and attribute counter from the attribute seed table, 2) encrypts the record with the corresponding attribute seed, and 3) insert the encrypted record to the Cloud.

DELETE: To delete an encrypted record from the outsourced database, it first retrieves the rowID of the records being deleted to update the attribute seed table before deleting the entries. The procedure involves three steps: 1) select the rowID of the records being deleted from the outsourced database, 2) update the attribute seed tables by incrementing the counter value of the corresponding rowID, and 3) delete the records from the outsourced database.

UPDATE: Updating the record can be achieved by deleting the old record and inserting the updated record. This contains the following procedures: 1) retrieve the records that needs to update, 2) delete the records from the outsourced database, 3) update the attributes locally, 4) re-encrypt the records with an unused rowID and attribute counter, and 5) insert the updated and encrypted records.

After each of these operations, the attribute seed table in the Cloud has to synchronize with the one at the user side so that the DBMS in the cloud server can have the most updated attribute seed for query execution. This attribute seed management can be automated by a software layer, which consists of 234 LOCs in our implementation prototype.

\section{Processor architecture}

In this section, we present our proposed CypherDB secure processor architecture that is capable of processing the encrypted data and protecting the query output.

Under our threat model, data must stay strongly encrypted outside the processor. Data confidentially is thus achieved if the processor only fetches (or stores) encrypted data from (or to) off-chip memory. The operating mechanism of our proposed processor involves three steps: 1 ) decrypts encrypted data loaded into the processor; 2) performs the specified database operation; 3) re-encrypts the result of the database operation before storing to off-chip memory. Such on-chip decryptprocess-encrypt operation has to be performed on every off-chip processor-to-memory data transaction. A simple database operation such as Query 1 in TPC-H [13] can result in millions of off-chip memory transactions. As a result, our secure processor architecture must be able to handle such off-chip memory transaction efficiently.

Another challenge in designing the CypherDB processor architecture is to minimize the software modification for deployment consideration. Having the data encrypted, the DBMS now has to handle the ciphertext rather than plaintext in our proposed architecture. The difficulty is that any intermediate value of the database operation stored off-chip also needs to be encrypted. This 


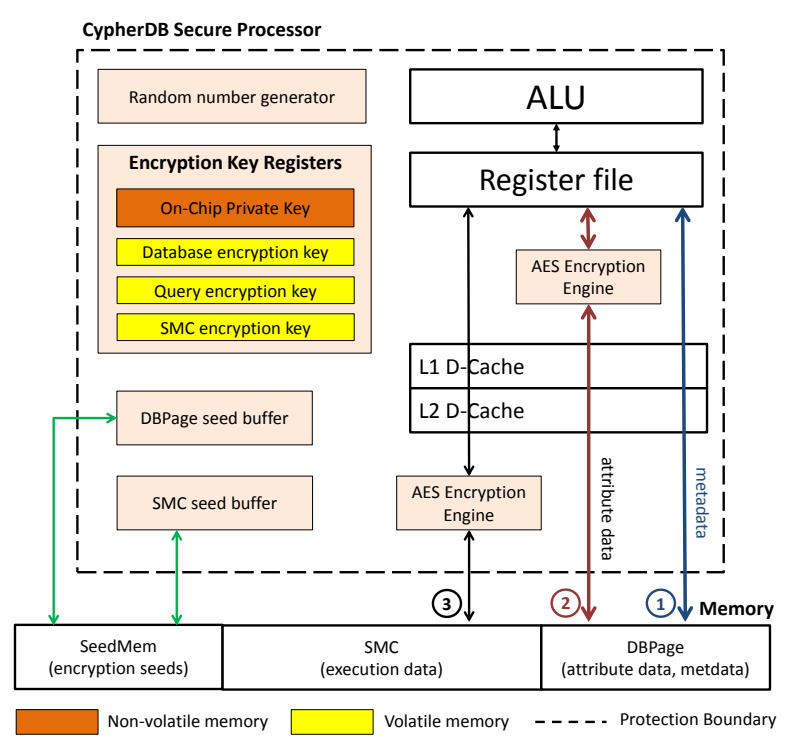

Fig. 5: Proposed architectural model of the secure processor.

requires a non-trivial modification of several operation layers in DBMS. The issue of minimizing such modification without incurring additional processing penalty is a challenging problem.

\subsection{Design Overview}

The key idea behind the CypherDB architecture in addressing the above challenges is to deploy dedicated hardware data paths to separately handle the three different types of data commonly found in any database application. In general, all data in a database application can be classified into three types: 1) attributes data, 2) metadata and 3) execution data. Attribute data represents the data from the database. Metadata includes nonsensitive information such as page header, record offset that is useful for the DBMS to manage the storage or access of the database records. Execution data is the intermediate value generated on-the-fly during program execution stored in heap or stack memory.

These three different types of data are stored in different locations on off-chip memory, which are designated as "compartments". Each memory compartment has its own data path to load its data into the processor securely and efficiently. Using our techniques, the DBMS only needs to explicitly handle the encrypted attribute data whereas the execution data is protected transparently by a specific hardware protection circuit. Thus, the major software modification can be confined to the data access layer only.

\subsection{Architectural Overview}

Figure 5 depicts the architectural model of the CypherDB processor. Off-chip memory is divided into three parts: seed storage compartment (SeedMem) for storing encryption seeds, secure memory compartment (SMC) for storing execution data, and data page compartment (DBPage) for storing attribute data and metadata. There are three data paths to execute these three types of data. AES encryption engines are used on two data-paths to process the encrypted database data and protect the memory content in SMC.

Four new special registers are designed to store the encryption keys. On-chip private key is written into the on-chip non-volatile register during chip manufacturing. There is no instruction support to modify or extract this key from the register. For the other three encryption keys, there is only instructions support to store the keys into these on-chip registers but without providing any instruction to extract them out. Database and query encryption keys can be dynamically stored into the on-chip registers with special instruction. SMC encryption key is generated by the on-chip random number generator at the beginning of the secure query execution.

DBPage and SMC seed buffers are on-chips memory used to store the encryption seeds in AES-CTR and AESOFB encryption. The purpose of these on-chip buffers is to reduce the off-chip memory access on fetching the seed for encryption.

\subsection{New Instructions Support}

The new instructions and their functionalities to support CypherDB is summarized in Table 1. During the key exchange between the database owner and the cloud servers, the database encryption key and query encryption key are stored into the on-chip registers via the load_encKey instruction. verify_encKey is used to verify the digital signature. To start the query execution, start_cypherdb_query is executed to allocate the SMC memory, generate a per-query encryption key by the random number generator and store it in the on-chip memory. switch_encKey is used to switch the encryption key between database encryption key and query encryption key along data-path (2). To end the query execution, end_cypherdb_query is executed to free the SMC memory and destroy the SMC encryption key.

TABLE 1: New Instructions in CypherDB.

\begin{tabular}{|l|l|}
\hline New Instructions & Description \\
\hline \hline start_cypherdb_query & $\begin{array}{l}\text { Allocate SMC and generate SMC en- } \\
\text { cryption key }\end{array}$ \\
\hline end_cypherdb_query & $\begin{array}{l}\text { Dis-allocate SMC and destroy SMC en- } \\
\text { cryption key }\end{array}$ \\
\hline mld & $\begin{array}{l}\text { Load attribute seed for attribute decryp- } \\
\text { tion/encryption }\end{array}$ \\
\hline secure_load, secure_store & $\begin{array}{l}\text { Decrpyt/encrypt attribute data with the } \\
\text { encryption pad }\end{array}$ \\
\hline load_encKey & $\begin{array}{l}\text { Load the encrypted database key and } \\
\text { encrypted query key; and decrypt the } \\
\text { keys with private key and store in on- } \\
\text { chip registers }\end{array}$ \\
\hline switch_encKey & $\begin{array}{l}\text { Switch the encryption key used in the } \\
\text { AES encryption engine along data-path } \\
2 \text { between database key and query key }\end{array}$ \\
\hline verify_encKey & $\begin{array}{l}\text { Verify the database key and query } \\
\text { key by checking the digital signature } \\
\text { Sig }\left(E_{P K}\left(K_{d b}, K_{Q}\right)\right)\end{array}$ \\
\hline
\end{tabular}




\subsection{Data Path Design}

In order to evaluate the performance impact of the three types of data accesses during a database query operation, we investigated the off-chip memory access profile, which is reported as having the most impact on execution time when performing database query [14]. Our investigation is based on executing the 22 queries in TPC-H [13] on SQLite using a cycle accurate simulator, Simplescalar [15], which resulted in three observations:

- Almost half of last-level data cache misses are caused by loading the metadata.

- Execution data has a high data reuse profile on the last-level cache - only less than $0.5 \%$ of them contribute to the last-level data cache misses.

- Last-level data cache misses on loading the attribute data varies from $2 \%$ to $34 \%$.

In view of the above observations, the design of CypherDB processor is focussed on providing three different data paths to handle these three types of data according to the aforementioned characteristics. The three data paths are (Figure 5) :

- Data-path (1): It is an unmodified data path akin to that used in a conventional processor to process the non-sensitive metadata. There is no processing penalty caused by this data path.

- Data-path (2): The attribute data is executed on this data path. Software handling is needed to perform on-chip decryption/encryption on the attribute data. As registers are the only software controllable on-chip memory, the encryption/decryption engine is therefore placed between the level 1 data cache and the register files.

- Data-path (3): All execution data has to follow this data path where the encryption engine is placed closest to the chip boundary due to its high last level data cache hit characteristic. The encryption or decryption is done on the entire cache line which is completely transparent to the application software.

To optimize performance, the off-chip encryption seeds are loaded into two separate on-chip buffers, DBPage seed buffer and SMC seed buffer, to encrypt/decrypt the attribute data and execution data respectively.

\subsection{Secure Access on Attribute Data}

One major disadvantage of this decrypt-process-encrypt operation is that the encryption and decryption operation is on the memory access critical path which is proven to be the performance bottleneck for a database system [14], [16]. Even if an optimized symmetric block cipher like AES is used, memory access latency to offchip memory would increase by over 30\% [17]. Using block cipher to encrypt the data also requires non-trivial handling of the encrypted data in software because the ciphertext has to be loaded into the processor in a fixed block size.

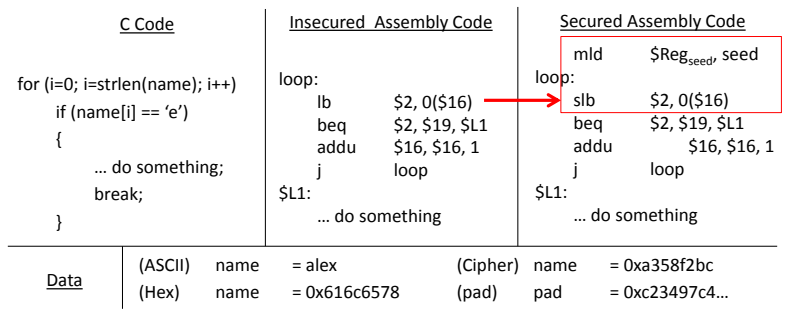

Fig. 6: An example comparing the code generated from a $\mathrm{C}$ code segment for an insecure processor and for the CypherDB secure processor

This shortcoming is alleviated through the use of AESCTR and AES-OFB as discussed in Section 3.1. Their impacts on the processor design are described as follows:

1. Latency offloading: Prior to loading the attribute data for processing, the attribute seed can be "generated" during the program execution. Through pre-computing the encryption pad (line 4 and line 15 in Algorithm 1), the actual data encryption/decryption latency is thus reduced to simple XOR operations (line 5 and line 19 in Algorithm 1). The encryption pads are generated automatically for AES-CTR and AES-OFB using the hardware AES encryption engines while the software is only responsible for triggering the encryption.

2. Stream Encryption/Decryption: Encrypting the data in streaming fashion simplifies the code generation task for accessing the encrypted attribute data because the data manipulations on attribute data are often byte, instead of block, oriented. Padding of byte-wide attribute data is thus not necessary, unlike the use of direct AES encryption.

This two properties are best illustrated using an example as shown in Figure 6. This can be seen, a code segment in $C$ searches for the letter ' $\mathrm{e}$ ' in a string variable name and then performs some database operations. The variable name is a data container for storing an attribute of VARCHAR type. Consider the case where name = "alex", which is stored as a 32-bit string 0x616c6578. Without using secure processing, the assembly code generated for the $\mathrm{C}$ construct is shown with typically four instructions. In order to use our CypherDB secure processor, the string name is stored in an encrypted form as cipher text $0 x a 358 \mathrm{f} 2 \mathrm{bc}$. This needs to be decrypted using the 128-bit pad value 0xc23497c4..... which has been pre-computed. In this case, the secure code segment only requires the $l b$ (load-byte) instruction be replaced by a two-instruction sequence: the mld instruction to load the seed of the corresponding attribute into the special register $R e g_{\text {seed }}$ to pre-compute the encryption pad $p$, and the $s l b$ instruction to load the encrypted data from the database page buffer and perform on-chip decryption. Decryption is done seamlessly, by shifting the encryption pad accordingly, within the CypherDB processor one byte at a time in a streaming fashion. 


\subsection{Secure Memory Compartment}

The reason of having SMC memory region on data path (3) is to protect any temporary execution data which may compromise security. To prevent such information leakage, any execution variable derived from the attribute data must also be encrypted on-chip before storing it in off-chip memory. One proposed solution to solving this problem is to use the technique of information flow tracking [18], [19]. This method taints all the intermediate data that has implicit and explicit relationship with the encrypted data. Although this solution can effectively trace the data that would potentially leak the sensitive information, it is still challenging to put it into practice [20].

In CypherDB architecture, the execution data is stored in SMC where each individual cache line in this memory compartment is encrypted using a hardware protection circuit embedded in the cache controller. We assume SMC can be dynamically brought up by some existing hardware-assisted isolated execution solutions such as Iso-X [21] or Bastion [9].

Putting the encryption/decryption engine between the last level cache and the off-chip memory satisfies two requirements: 1) the overhead of encryption is incurred only when data is written back to external memory from the cache, thus reducing the performance overhead incurred by securing the execution data; 2 ) the protection mechanism is transparent to the database software, thus minimizing the requirement for software modifications.

\subsubsection{Cache Line Encryption}

For every memory transaction, each SMC cache line is decrypted/encrypted using AES-CTR when it is loaded into (or written back from) the processor. AES-CTR is used to offload the encryption penalty from the critical path by parallelizing the seed encryption and off-chip memory access of the cache line. Since the last level cache line usually consists of multiple 128-bit data blocks, multiple hardware encryption engines can be operated in parallel on those data blocks. Thus, the entire cache line can be decrypted/encrypted in a single cycle.

To ensure security, a unique seed is generated for each cache line for the entire SMC memory space. This unique cache line seed, CLSeed, is produced by concatenating two fields: 1) the tag portion of the physical memory address, and 2) a unique counter value count_val:

$$
\text { CLSeed }=\text { physical address tag } \| \text { count_val. }
$$

The count_val associated with each cache line is stored in off-chip memory, and is incremented every time the cache line is written back to off-chip memory. In this means, each cache line is uniquely encrypted and security is therefore guaranteed.

\subsubsection{Random Per-Query Key}

To encrypt or decrypt information, an encryption key is required in addition to the unique seed value. Cache line encryption using AES-CTR has been extensively studied in [22], [23]. However, these approaches use a single shared key to encrypt the entire memory space. This can lead to two significant drawbacks: 1) using a constant key introduces vulnerability to attacks; 2) in counter mode encryption, the counter could overflow and the count value would wrap around, thus compromising security because the seed value is no longer unique.

In CypherDB, for each query execution, a random key generated by the on-chip random number generator is used to encrypt the cache lines in SMC. This random key is overwritten when a new query is executed. The idea of employing such per-query random key is based on two major insights:

1) In database applications, a query is self-contained, and it is unnecessary to carry forward the execution data to another query.

2) Operation on the encrypted database is transparent to the user who is only interested in the result of the query. Consequently, the user does not need to have knowledge of the key used, and a random key would be suitable.

The advantages of using random per-query key to encrypt the data in SMC are three-fold. Firstly, it isolates the encryption protection on attribute data and on-thefly execution data by employing different encryption keys. Even if one can break the encryption in SMC, the most critical secret - the database encryption key $K_{d b}$, is still safe from tampering. Secondly, the uniqueness of the seed used in database encryption can remain "unpolluted" regardless of the execution in SMC. This makes the re-encryption of the attribute data with a new seed value become data-update dependent rather than program-execution dependent. Lastly, regenerating the encryption key for each query processing can refresh the uniqueness of the physical address and the incremental counter so as to reduce the re-encryption frequency.

\subsection{Query Output Protection}

After processing the query on the encrypted database, the query output has to be sent back to the user. Protection on the query output is necessary because it contains aggregation result or the actual data of the encrypted database.

The query output is protected by encrypting it in the same way as attribute encryption. This can be achieved because the result of the SELECT query can always be organized in a database table where the select-list right after the SELECT clause defines the columns of the table. For instance, the callback function in SQLite returns a row of the query output at a time, where the items in the select-list are stored in an array. Each item of the query output can therefore be encrypted by AES-CTR and AES-OFB, except the attribute seed is in the form of (queryID, rowID, columnID). The reason of using AESCTR and AES-OFB is the same as attribute encryption to facilitate the processing in our secure processor. 


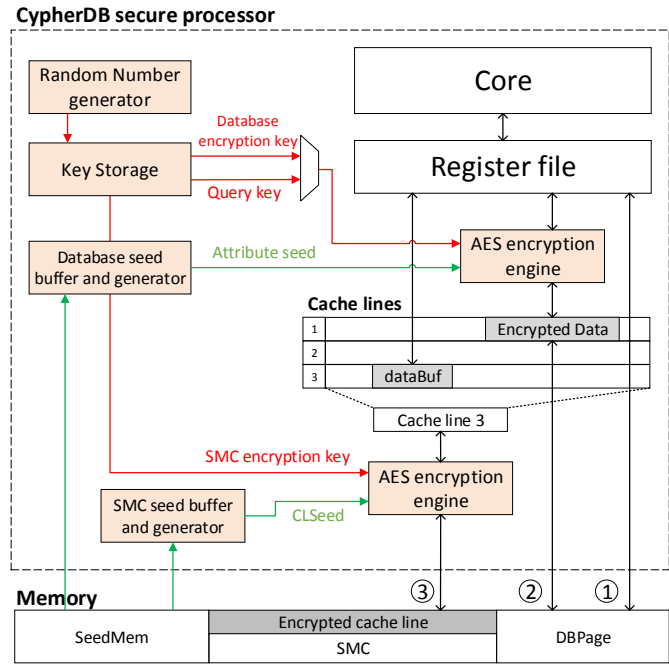

Fig. 7: The process of copying the encrypted data into a data buffer within SMC in hardware.

\subsection{Query Execution Flow}

In this section, we demonstrate how CypherDB secures query processing on the encrypted database with the use of the aforementioned three data-paths. For ease of illustration, we consider a query to be processed as "SELECT Name, Remark FROM table WHERE ID $O P E<$ 24675431" (see Figure 4).

In this query, the operation involves a $\mathrm{B}^{+}$tree search on the index $\left(I D_{O P E}\right)$, copying the relevant attributes (Name, Remark) to some temporarily variables (dataBuf in Figure 7) and returning back the query result (dataBuf) to the user. Figure 7 shows the data flow of this query operation in CypherDB.

Before the start of query execution, the DBMS follows 2 steps to set up the secure execution environment: 1) load and verify database and query encryption keys via load_encKey and verify_encKey instructions respectively, and 2) generate the SMC encryption key and setup SMC memory via start_cypherDB_query instruction. The database encryption key is set as the default encryption key along data-path (2).

The query execution starts by following data-path (1) to process some metadata and search for the index. Note that the index is encrypted with OPE such that no decryption is needed for the index search.

For a matched condition $\left(I D_{O P E}<24675431\right)$, the related encrypted attributed has to be processed along data-path (2). Before accessing the encrypted attribute, the seed value is first loaded into the seed register $R e g_{\text {seed }}$ via mld instruction. The encryption pad is generated using database encryption key with the value in Reg $g_{\text {seed }}$ and the attribute counter in DBPage seed buffer. To access the encrypted attribute, the encrypted data is loaded into the processor via secure load instruction.

The result of the operation, which is the data itself, has to be stored in SMC. Prior to storing the modified cache line (cache line 3) back to the off-chip memory, the whole cache line is encrypted with SMC encryption key
TABLE 2: Parameters used in simulations

\begin{tabular}{|c|c|}
\hline Parameters & Specifications \\
\hline \hline Processor Architecture & MIPS ISA \\
\hline L1 I/D cache & $\begin{array}{r}\text { Split, 32KB, 2-way, 32B blocks size, } \\
\text { 2 cycles latency }\end{array}$ \\
\hline L2 I/D cache & $\begin{array}{r}\text { Split, 2MB, 4-way, 64B blocks size, } \\
6 \text { cycles latency }\end{array}$ \\
\hline Load/Store queue size & 64 \\
\hline Register update unit size & 128 \\
\hline TLB & 4 -way, 128 entries \\
\hline Main Memory & 4GB, 200 cycles latency \\
\hline AES latency & 80 cycles \\
\hline
\end{tabular}

and an incremented counter value in SMC seed buffer together with the physical address along data path (3).

Eventually the data stored in dataBuf has to be returned back to the user as a query output. This has to be done in 3 steps: 1) load the encrypted cache line along data path (3); 2) switch the encryption key from $K_{d b}$ to $K_{Q}$ via switch_encKey instruction on data path (2); and 3) encrypt the query output using $K_{Q}$ and the query seed (see Section 4.7) along data path (2).

\section{Performance Evaluation}

In this section, the performance of CypherDB on encrypted database is compared with that without encryption. The total execution cycles are measured in both cases and the resulting slowdown percentage is recorded. CypherDB incurs performance overhead in two places: 1) extra instruction executions when copying the on-chip database seeds into $R e g_{\text {seed }}$ to perform onchip data decryption/encryption; 2) extra memory accesses when fetching the counter value of the attribute seeds or SMC seeds from off-chip memory. Our evaluation is conducted using a set of computation-intensive analytical workload on database which makes high demand to the cloud resources. The simulation framework and the selected workload are first described. We then present our experimental results with a case study based on a commercial DBMS.

\subsection{Simulation Framework}

We evaluate CypherDB architecture using the SimpleScalar tool set [15]. We model a speculative out-oforder processor with a two-level cache hierarchy with separate data and instruction caches. The parameters used in simulation are shown in Table 2.

In our experiments, a separate 16-bit seed bus is used to load the counter value of the tuple and SMC seeds into the corresponding on-chip buffers. The attribute and the SMC counter seeds, both 16-bit wide, are loaded from off-chip memory. Each time either seed value is reloaded to the on-chip buffer, latencies are incurred due to offchip memory access and the need to generate a new encryption pad value.

For each measurement, we execute the query twice. The first query is to fill the pipeline and the data page 
buffers in main memory. All performance measurement, including the query output protection, is only taken on the second query execution. In the simulations, we ignore the database/query encryption key exchange and random per-query key initialization overhead because it is negligible when compared to the steady-state performance.

\subsection{Workloads}

In our study, we first use a data access kernel to evaluate the performance impact of the on-chip decryption/encryption on data path (2). Our second experiment is to run a scaled down workload, DBmBench [24], on a real database system, SQLite [25], in order to study the effect of the cache line decryption/encryption engines (3). Finally, a comprehensive performance evaluation on different representative query executions is conducted with the use of the TPC-H [13] benchmark. In all experiments, the data set is generated by the TPC-H data generator and is encrypted with our database encryption method described in Section 3.

Data Access Kernel: Our data access kernel is configured to perform sequential scan and $\mathrm{B}^{+}$-tree nonclustered index scan on a $100 \mathrm{MB}$ data file which consists of $600 \mathrm{k}$ records. These two scan operators are chosen because they are the most dominant data access operators in any DBMS. They represent two major data access patterns: predominantly sequential (sequential scan) and predominantly random (non-clustered index scan) record access. We further compare the performance impact of AES-CTR in CypherDB with an alternative approach that uses conventional AES block cipher to perform the same on-chip decryption/encryption on the same data path.

DBmBench: DBmbench contains three representative queries that accurately mimics the well-known TPC database workload [24] at the micro-architecture level. These queries includes three dominating operators: 1) sequential scan (uSS); 2) index scan (uIDX) and; 3) join query (uNJ).

To compare the performance of CypherDB architecture with the same query execution over an encrypted database using homomorphic encryption, we create a homomorphically encrypted database using the same methodology in [4]: the database is encrypted with Order Preserving Encryption for range check, Pailliar Encryption for summation, blowfish for equality check. To construct a similar query execution environment over the homomorphically encrypted database in line with [4], an application program written in $\mathrm{C}$ is used to perform the aforementioned three representative queries using the unmodified SQLite libraries. To perform summation operation, gmp library is used to multiply the encrypted data.

TPC-H: We evaluate the performance of CypherDB over the 22 queries in TPC-H. In our experiments, the evaluation is performed on a $100 \mathrm{MB}$ dataset, which is the
TABLE 3: The Slowdown Percentage of CypherDB and direct AES with our data access kernel on sequential scan and nonclustered index scan.

\begin{tabular}{|c|c|c|c|}
\hline & & CypherDB & Direct AES \\
\hline \multirow{3}{*}{ Sequential Scan } & sum(1) & $44 \%$ & $103 \%$ \\
& $\operatorname{sum}(2)$ & $44 \%$ & $116 \%$ \\
& $\operatorname{sum}(4)$ & $49 \%$ & $160 \%$ \\
\hline \multirow{3}{*}{ Non-Clustered Index Scan } & $\operatorname{sum}(1)$ & $25 \%$ & $27 \%$ \\
& $\operatorname{sum}(2)$ & $25 \%$ & $28 \%$ \\
& $\operatorname{sum}(4)$ & $25 \%$ & $29 \%$ \\
\hline
\end{tabular}

largest volume of data that can be process in reasonable time using our simulation environment.

\subsection{Performance on Data Access Kernel}

In our experiments, a dedicated function $\operatorname{sum}(n)$ is used to add $n$ attributes within a record together. Such summation operation is performed on $20 \%$ of the records. In our implementation, every off-chip memory access on an attribute seed fetches a block of seed counter value via the 16-bit seed bus. Based on our investigation (see Appendix A), it is found that the DBPage seed buffer of 8 bytes block size, which can accommodates four attribute seed counter value, is most efficient in both sequential and random record access.

Table 3 compares the performance of CypherDB approach with a direct AES encryption approach. CypherDB executes additional instructions of around $20 \%$ on sequential scan and $1.2 \%$ on index scan. Using direct AES encryption introduces on-chip decryption latency for loading every encrypted data. As each attribute is encrypted in 128-bit cipher block, the decrypted data used up more space in cache memory, resulting in extra off-chip memory accesses to fetch the data. It results in $160 \%$ slowdown while CypherDB has a steady performance of around $45 \%$ for sequential scan operator. This comparison shows that AES-CTR, in addition to its software-friendly characteristic, performs better than a direct AES approach in terms of performance.

\subsection{Case Study on SQLite}

In order to evaluate CypherDB on a complex system, we run the benchmark DBmBench and the well-known benchmark TPC-H on SQLite. We modify the file access layer and incorporate the DBPage seed into the software execution as discussed in Section 3 and Section 4 accordingly. The modified file access layer (see Appendix D) consists of 208 additional LOCs.

\subsubsection{Performance on DBmBench}

As SMC seed buffer is to store the counter value for cache-line encryption, its implementation is akin to an conventional cache architecture. According to our investigation (see Appendix B), a SMC seed buffer with 8 bytes block size, fully associative, and with leastrecently-used replacement policy yields the best performance. 


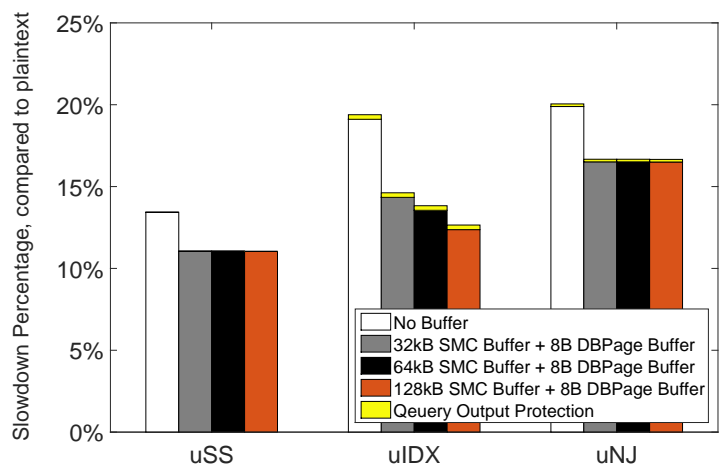

Fig. 8: Performance evaluation of CypherDB with different SMC buffer size on DBmBench.

TABLE 4: Queries executed instruction overhead in DBmBench

\begin{tabular}{|c|c|c|c|}
\hline & uSS & uIDX & uNJ \\
\hline Overhead percentage & $12 \%$ & $19 \%$ & $37 \%$ \\
\hline
\end{tabular}

Based on this architecture, we evaluate the performance impact of CypherDB with SMC buffer size ranges from $32 \mathrm{kB}$ to $128 \mathrm{kB}$ as shown in Figure 8 . Without any on-chip DBPage or SMC buffer, the performance overhead on DBmBench is around $17 \%$ on average, ranging from $13 \%$ to $20 \%$. The performance overhead is not significantly higher even without on-chip buffer because our data path design introduces no processing penalty on the execution of the non-sensitive metadata, which is proven as the culprit of most data stalls in our benchmark profiling. To achieve a better performance, on-chip buffers can significantly reduce the performance slowdown of uIDX query from $20 \%$ to $12 \%$. A slight performance improvement can also be observed on uSS and $\mathrm{uNJ}$ queries by reducing $2 \%$ and $3 \%$ slowdown percentage respectively. Less than $0.3 \%$ slowdown is attributed to the query output encryption for all queries.

Table 4 shows the executed instruction overhead on the three queries in DBmBench, as introduced by copying the attribute seed into $R e g_{\text {seed }}$. The instruction overhead of $\mathrm{uNJ}$ is relatively high because the execution involves nested loop join on two tables. Extra instructions are used to copy the corresponding tableID of the attribute seed on this query execution. In contrast, uSS and uIDX execution involves one table only, therefore has less instruction overhead than uNJ execution.

\subsubsection{Performance Comparison with Homomorphically Encrypted Database}

TABLE 5: Slowdown Percentage of CypherDB with 128kB SMC Buffer and the execution over a homomorphically encrypted database on DBmBench.

\begin{tabular}{|c|c|c|}
\hline & CypherDB & Homomorphic Encryption \\
\hline uSS & $11 \%$ & $52 \%$ \\
\hline uIDX & $12 \%$ & $1333 \%$ \\
\hline uNJ & $17 \%$ & $705 \%$ \\
\hline
\end{tabular}

We further compare the performance of CypherDB architecture with the execution on a homomorphically encrypted database. Our evaluation, as shown in Table 5, shows that the execution over a homomorphically encrypted database suffers from a significant slowdown ranging from $52 \%$ on uSS to $1333 \%$ on uIDX. The slowdown on uSS is minimal because only equality and range check are performed in this query, where blowfish and OPE encryption are very efficient on these operations respectively. In spite of their efficiency on these operations, blowfish and OPE encryption transform any data less than 64 bits into a 64-bit cipher. It thus induces extra execution overhead on handling the enlarged data throughout the DBMS execution, resulting in a moderate performance overhead on uSS execution. On the other hand, uIDX and uNJ both involves summation, which requires the multiplication of two 2048-bit Pailliar encrypted data. By transforming a 32-bit integer summation function into a multiplication operation of two 2048-bit data, the execution cycles of uIDX and uNJ increases by $14 \times$ and $8 \times$ respectively. CypherDB, on the contrary, maintains a steadily reasonable performance overhead of $11 \%$ to $17 \%$, regardless of the operations involved.

\subsubsection{Performance on TPC-H}

Figure 9 illustrates the performance of CypherDB on TPC-H queries. The average performance overhead is only $10 \%$ ranging from $3 \%$ for query 11 to $23 \%$ for query 14 . The query output encryption results in $0.35 \%$ slowdown on average. We also evaluate the instruction count overhead of the 22 queries in TPC-H as summarised in Table 6. On average, there is around $14 \%$ more instructions being executed in our proposed solution, ranging from $8 \%$ in query 12 to $28 \%$ in query 14. This instruction overhead is the main contributor to the performance degradation. CypherDB increases the database size by $28 \%$, of which $21 \%$ comes from the OPE encrypted indices. The remaining $7 \%$ is because the encrypted data disable the compression in SQLite.

\subsection{Evaluation on FPGA Platform}

To have a more practical evaluation environment, we implemented our CypherDB secure processor, particularly data-path (1) and data-path (2), on a Field-Programmable Gate Array (FPGA) platform. We built our CypherDB secure processor architecture based on OpenRISC [26], an open source RISC soft-processor. We also employ the open source AES encryption engine from OpenCore [26]. Our hardware implementation is specified in Verilog. It is synthesized and implemented using ALTERA Quartus 14.0 design tool targeting the DE2i-150 board with a Cyclone IV SoC FPGA. The modified SQLite-3.6.14 is running on top of Linux-3.13.

DBmBench is used to evaluate the hardware implementation of CypherDB secure processor. Our evaluation metrics are based on the timing function built in SQLite 


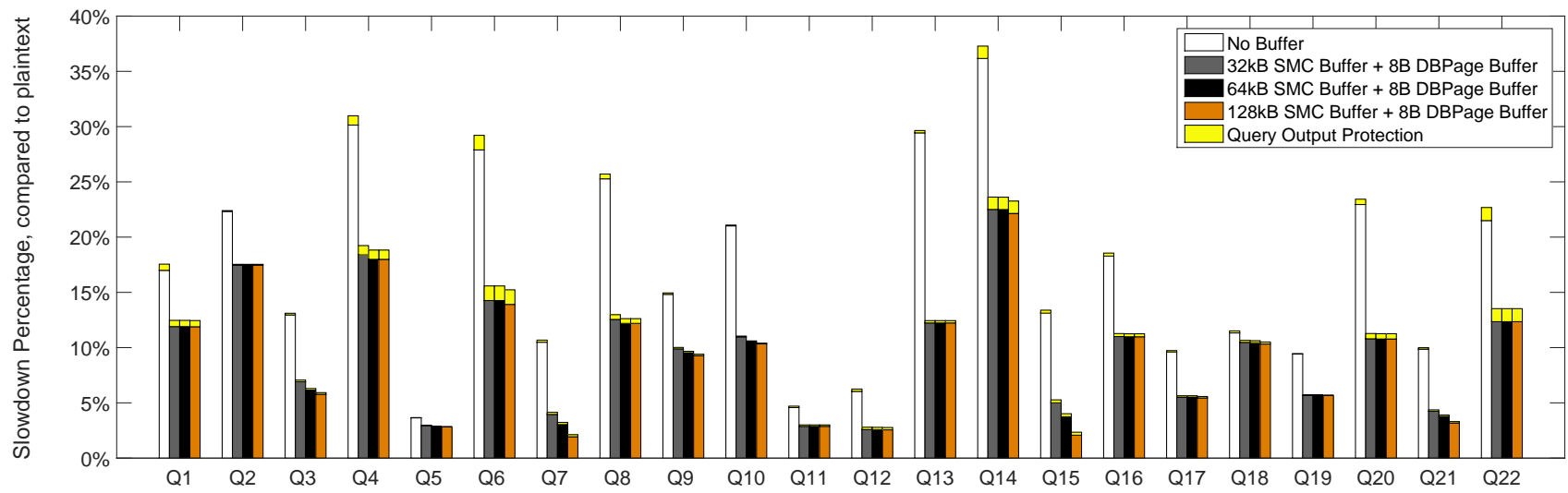

Fig. 9: Performance evaluation of CypherDB with various SMC buffer size on the 22 queries in TPC-H.

TABLE 6: Queries executed instruction overhead in TPC-H

\begin{tabular}{|c|c|c|c|c|c|c|c|c|c|c|c|}
\hline & Q1 & Q2 & Q3 & Q4 & Q5 & Q6 & Q7 & Q8 & Q9 & Q10 & Q11 \\
\hline Overhead percentage & $10 \%$ & $17 \%$ & $12 \%$ & $19 \%$ & $14 \%$ & $14 \%$ & $11 \%$ & $17 \%$ & $11 \%$ & $15 \%$ & $11 \%$ \\
\hline & Q12 & Q13 & Q14 & Q15 & Q16 & Q17 & Q18 & Q19 & Q20 & Q21 & Q22 \\
\hline Overhead percentage & $8 \%$ & $14 \%$ & $28 \%$ & $13 \%$ & $13 \%$ & $13 \%$ & $19 \%$ & $9 \%$ & $21 \%$ & $10 \%$ & $16 \%$ \\
\hline
\end{tabular}

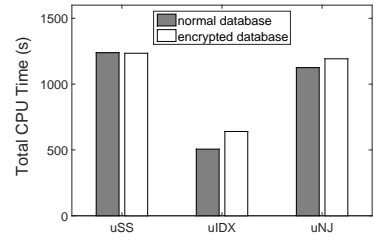

(a) 500MB database

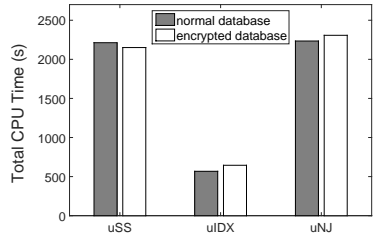

(b) 1GB database
Fig. 10: Total CPU time spent on running the DBmBench query benchmark on (a) $500 \mathrm{MB}$ and (b) 1GB database size.

which can report the total CPU time taken during query execution. The accurate timing information is provided by a hardware tick timer implemented in FPGA. In all experiments, each query was ran 5 times and the average CPU runtime is recorded. We ran the queries on $500 \mathrm{MB}$ and 1GB database which comprises of around 4 and 8 millions records, respectively.

Figure 10 shows the total CPU time spent on processing the queries on $500 \mathrm{MB}$ and $1 \mathrm{~GB}$ database. It is found that the performance overhead caused by the on-chip decryption along data-path (2) is negligible in uSS and uNJ due to our careful hardware design. uIDX shows a modest performance penalty ranging from $14 \%$ to $26 \%$. To investigate the reason of these performance overhead, we analyzed the CPU time spent on user codes and kernel codes separately. Our investigation found that the CPU time spent on user code ranges from $1 \%$ to $11 \%$. These overhead is caused by the extra instructions since they were inserted in user code space. On the other hand, the CPU time spent on kernel code ranges from $20 \%$ to $43 \%$. It is because the record counters occupied a small portion of the physical memory. With the reduction of the available memory, more time is spent on kernel to allocate and free memory for the query execution. This overhead is observable due to an experimental limitation that only a small memory $(64 \mathrm{MB})$ is available in our FPGA evaluation platform. It would be alleviated with the use of a larger memory.

\section{Security Analysis}

We now evaluate the security of CypherDB system. Our discussion is focused on protecting confidentiality of the data because the goal of CypherDB is to prevent information leakage through passive attack.

\subsection{Database Security}

The security of AES-CTR and AES-OFB is well-proven [27], except that they pose a strong requirement on the encryption seed - must be unique for each datum under a single encryption key. Otherwise, the confidentiality of the data may be compromised due to the "two-time" pad attack caused by re-using the same encryption pad. In our proposed encryption scheme, each attribute seed is spatially and temporally unique across the databases for the same database owner, as described in Section 3.2. Various database owners have their own unique database encryption keys $K_{d b}$ such that the seed uniqueness concern is confined to a single party. It therefore greatly simplifies the attribute seed management and is relied on the CypherDB supported software to handle the seed uniqueness. Re-encrypting the database with a new encryption key may be necessary when the attribute seeds, either the logical schema ID or tuple counters, overflows. These parameters are set to a sufficient large value to avoid frequent re-encryption.

CypherDB employs three different encryption keys for various encryption purposes. The database encryption key is therefore isolated for the ease of maintaining the 
seed uniqueness. The use of these encryption modes however provides some security strength. It is because the encrypted data are non-deterministic due to the unique encryption seed used. It means that even two attributes are of the same value, the encrypted data looks completely different.

CypherDB does not stress on encrypting the query statements. One may concern the information leakage from the query statements. We refer it as an indirect information leakage. Take a query statment like "SELECT NAME, AGE FROM TABLE WHERE ID='1234';" as an example. An adversary can learn from this unencrypted query statement that the user is searching for NAME and AGE with a certain ID number. However, he is unable to learn the exact information because all the query outputs are encrypted. The corresponding information leakage is at most the number of entries satisfying the WHERE clause. Such indirect information leakage can be solved by sending dummy output result but it is not considered in this paper.

\subsection{System Security}

CypherDB protects all sensitive data storing in off-chip memory with strong encryption. Breaking the encryption is made infeasible by not revealing the encryption key and using a unique seed value for database encryption and cache line encryption.

Potential attack can be launched by impersonating the database owner to query the encrypted database. For example, an adversary can impersonate the database owner to issue a query with his own query key. In this attack, the adversary can make use of the "empowered" CypherDB processor to learn the sensitive data, even though the encryption key is not disclosed. However, this is prohibited because the digital signature of each query key can verify the owner of the query key. The query key is stored in on-chip register without revealing after verification. The adversary is unable to impersonate or read the query key.

To crack the database encryption, the adversary can send a wrong seed value to the seed register in order to perform "two-time-pad" attack. This is however out of scope in this paper because such attack has to alter the program execution which is protected by [10]. Also, it will also result in an incorrect database operation, which violates our curious-but-honest administrator threat model.

\section{Related Work}

Many solutions have been proposed to secure database outsourcing. CryptDB [4] uses SQL-aware encryption to support confined set of operations heavily used in database but fails to address analytical operations. Split client-server [28] execution approach allows approximate filtering at the server and compute the final query result at the client which is impractical when dealing with big data on a resource-constrained client computing device. The approach in [5] combines the above methods but requires careful modification of the query and demonstrates up to $2 \times$ performance slowdown. Alternatively, TrustedDB [6] permits query execution entirely inside a secure co-processor but introduces almost $8 \times$ slowdown.

The decrypt-process-reencrypt style operation involved in our CypherDB processor design has been studied and used in commercial product. TPM [8] can perform on-chip encryption/decryption by using a sealed key stored inside the chip. However, it offers no protection to the decrypted data once it leaves the chip. Passive reading on the memory is thus made possible. Cipherbase [7] uses a trusted FPGA, acting as a co-processor, to perform decrypt-process-reencrypt database operation by decomposing the database operations at the operator level. However, the communication between the server and FPGA becomes another bottleneck on large database processing. CypherDB is the first to use this decrypt-process-reencrypt sytle operation on the processor execution model.

Re-designing the processor architecture to encrypt the off-chip memory was also extensively studied in [22], [23], [29], [30]. These approaches use solely hardware mechanism to encrypt the entire off-chip memory, including the code and data, as a whole. This heavily prohibits database virtualization in the cloud because re-encryption is required when the data migrates from one storage/computational node to another. CypherDB, which encrypts the database in its logical schema, enables the virtualization through a combined software and hardware design.

\section{Conclusions}

This paper presents a novel processor architectural design to perform secure and efficient query processing on an encrypted database. With minimal modifications to the database application software, our proposed processor architecture, CypherDB, can achieve a higher security and performance efficiency when compared with solutions using homomorphic encryption or trusted coprocessor. Our simulation results show that it introduces on average $10 \%$ performance overhead and $14 \%$ executed instruction count overhead and $28 \%$ storage overhead. Further reduction in the performance and instruction count overhead may be possible via register sharing of the attribute seed and the program execution.

Our work is being extended in several directions. One interesting direction would be to incorporate our system into an In-Memory database environment, which potentially is more efficient in accessing data. Another direction relates to the use of vector processing in the modern processor systems.

\section{References}

[1] K. Getgen, “2009 encryption and key management industry benchmark report: A risk management benchmark for data protection," Trust Catalyst. 
[2] "Google find employees for breaching user priCNET News, 2010. [Online]. Available: http://www.cnet.com/uk/news/google-fired-engineer-forprivacy-breach/

[3] C. Gentry, S. Halevi, and N. P. Smart, "Homomorphic evaluation of the AES circuit," Cryptology ePrint Archive, Tech. Rep., June 2012.

[4] R. A. Popa, C. M. S. Redfield, N. Zeldovich, and H. Balakrishnan, "CryptDB: Protecting Confidentiality with Encrypted Query Processing," in Proceedings of the Twenty-Third ACM Symposium on Operating Systems Principles, ser. SOSP '11, 2011, pp. 85-100.

[5] S. Tu, M. F. Kaashoek, S. Madden, and N. Zeldovich, "Processing Analytical Queries over Encrypted Data," in Proceedings of the $V L D B$ endowment, Aug 2013.

[6] S. Bajaj and R. Sion, "TrustedDB: a trusted hardware based database with privacy and data confidentially," in Proceedings of SIGMOD, June 2011, pp. 205-216.

[7] A. Arasu, S. Blanas, K. Eguro, R. Kaushik, D. Dossmann, R. Ramamurthy, and R. Venkatesan, "Orthogonal security with $\mathrm{Ci}$ pherbase," in Proceedings of the 6th CIDR, Jan 2013.

[8] "Trusted computing group." [Online]. Available: http://www.trustedcomputinggroup.org

[9] D. Champagne and R. Lee, "Scalable architectural support for trusted software," in High Performance Computer Architecture (HPCA), 2010 IEEE 16th International Symposium on, Jan 2010, pp. $1-12$.

[10] E. Aktas, F. Afram, and K. Ghose, "Continuous, low overhead, run-time validation of program executions," in Microarchitecture (MICRO), 2014 47th Annual IEEE/ACM International Symposium on, Dec 2014, pp. 229-241.

[11] M. Dworkin, "Recommendation for block cipher modes of operation," National Institute of Standards and Technology, Tech. Rep., 2001.

[12] R. A. Popa, F. H. Li, and N. Zeldovich, "An ideal-security protocol for order-preserving encoding," in Proceedings of the 2013 IEEE Symposium on Security and Privacy, ser. SP '13. Washington, DC, USA: IEEE Computer Society, 2013, pp. 463-477.

[13] "The tpc-h benchmark." [Online]. Available: http://www.tpc.org/tpch/.

[14] A. Ailamaki, D. J. DeWitt, M. D. Hill, and D. A. Wood, “DBMSs on a modern processor: where does time go?" in Proceedings of International Conference on Very Large Databases, 1999.

[15] T. Austin, E. Larson, and D. Ernst, "Simplescalar: An infrastructure for computer system modeling," Computer, vol. 35, no. 2, pp. 59-67, Feb. 2002.

[16] P. A. Boncz, S. Manegold, and M. L. Kersten, "Database architecture optimized for the new bottleneck: Memory access," in Proceedings of the 25th International Conference on Very Large Data Bases, ser. VLDB '99, 1999, pp. 54-65.

[17] S. Gueron, "Intel advanced encryption standard (aes) new instructions set," Intel Corporation, Tech. Rep., May 2010.

[18] G. E. Suh, J. W. Lee, D. Zhang, and S. Devadas, "Secure program execution via dynamic information flow tracking," in Proceedings of the 11th International Conference on Architectural Support for Programming Languages and Operating Systems, ser. ASPLOS XI, 2004, pp. 85-96.

[19] Y.-Y. Chen, P. A. Jamkhedkar, and R. B. Lee, "A software-hardware architecture for self-protecting data," in Proceedings of the 2012 ACM Conference on Computer and Communications Security, ser. CCS '12, 2012, pp. 14-27.

[20] S. Zdancewic, "Challenges for information-flow security," in In Proc. Programming Language Interference and Dependence (PLID, 2004.

[21] D. Evtyushkin, J. Elwell, M. Ozsoy, D. Ponomarev, N. Abu Ghazaleh, and R. Riley, "Iso-x: A flexible architecture for hardwaremanaged isolated execution," in Microarchitecture (MICRO), 2014 47th Annual IEEE/ACM International Symposium on, Dec 2014, pp. 190-202.

[22] J. Yang, Y. Zhang, and L. Gao, "Fast secure processor for inhibiting software piracy and tampering," in Proceedings. 36th Annual IEEE/ACM International Symposium on Microarchitecture, 2003. MICRO-36., 2003, pp. $351-360$.

[23] G. E. Suh, C. W. OD́onnell, and S. Devadas, "Aegis: A single-chip secure processor," in Design $\mathcal{E}$ Test of Computers, IEEE, vol. 24, 2007 , pp. $570-580$
[24] M. Shao, A. Ailamaki, and B. Falsafi, "Dbmbench: Fast and accurate database workload representation on modern microarchitecture," in Proceedings of the 2005 Conference of the Centre for Advanced Studies on Collaborative Research. IBM Press, 2005, pp. 254-267.

[25] "Sqlite homepage." [Online]. Available: http://www.sqlite.org/.

[26] "Opencores community." [Online]. Available: http:/ /opencores.org

[27] T. G. Wayne Jansen, "Guidelines on security and privacy in public cloud computing," National Institute of Standards and Technology, Tech. Rep., Dec 2011.

[28] H. Hacigümüş, B. Iyer, C. Li, and S. Mehrotra, "Executing sql over encrypted data in the database-service-provider model," in Proceedings of the 2002 ACM SIGMOD International Conference on Management of Data, ser. SIGMOD '02. ACM, 2002, pp. 216-227.

[29] B. Rogers, S. Chhabra, Y. Solihin, and M. Prvulovic, "Using address independent seed encryption and bonsai merkle trees to make secure processors os- and performance-friendly," in Proceedings of the 40th Annual IEEE/ACM International Symposium on Microarchitecture (MICRO 40), 2007, pp. 183 - 196.

[30] D. Lie, C. Thekkath, P. Lincoln, M. Mitchell, D. Boneh, J. Mitchell, and M. Horowitz, "Architectural support for copy and tamper resistant software," in Proceedings of the ninth international conference on Architectural support for programming languages and operating systems (ASPLOS-IX), 2000, pp. 168 - 177.

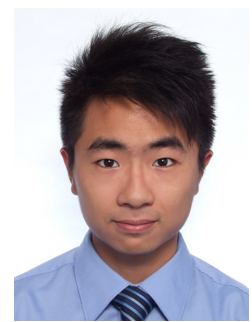

Bony H. K. Chen received his BEng in electronic engineering from the University of Hong Kong in 2011. He is currently working toward the Joint $\mathrm{PhD}$ degree at the Department of Electrical and Electronic Engineering of the University of Hong Kong and Imperial College London. His research interests include cloud computing security, database security and hardware-enhanced security.

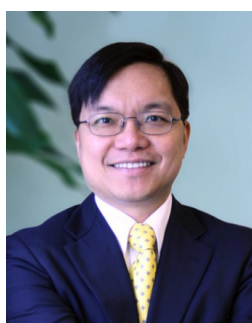

Paul Y. S. Cheung is Associate Vice-President (Research) and Professor in Electrical and Electronic Engineering at University of Hong Kong. He received his B.Sc.(Eng) and Ph.D. degrees from Imperial College London in 1973 and 1978 respectively. He joined the University of Hong Kong in 1980 and served as Dean of Engineering from 1994-2000. He was on IEEE AsiaPacific Director (1995-96) and IEEE Secretary (1997). He was Chair of IEEE Medal of Honor Committee (2009-12), Chair of IEEE Awards Board (2007-08) and a Director of IEEE Foundation (2008-14).

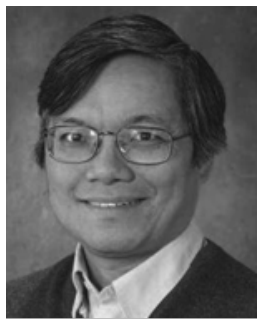

Peter Y. K. Cheung received the B.S. degree with first class honors from Imperial College of Science and Technology, University of London, London, U.K., in 1973. Since 1980, he has been with the Department of Electrical Electronic Engineering, Imperial College London, where he is currently a Professor of digital systems, Vice Dean (Education) in the Faculty of Engineering and and Head of the EEE department. He runs an active research group in reconfigurable circuits and systems. His research interests include VLSI architectures for signal processing, asynchronous systems, reconfigurable computing using FPGAs, and architectural synthesis.

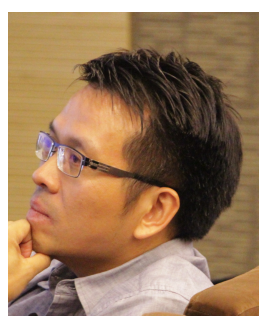

Yu-Kwong Kwok (Ricky) is Associate VicePresident (Teaching and Learning) and Professor in the Electrical and Electronic Engineering Department at the University of Hong Kong $(\mathrm{HKU})$. He received his B.Sc. degree in computer engineering from HKU in 1991, the M.Phil. and $\mathrm{Ph}$.D. degrees in computer science from the Hong Kong University of Science and Technology (HKUST) in 1994 and 1997, respectively. Ricky has been an Associate Editor for the IEEE Transactions on Parallel and Distributed Systems since January 2013. He is a Fellow of the HKIE, the IEEE, and the IET. He is also a member of the IEEE Computer Society and the IEEE Communications Society. 